\title{
Modelagem Genética para Estender o Tempo de Vida de Redes de Sensores Sem Fio com Sorvedouro Móvel*
}

\author{
Sérgio de Oliveira, Pedro Mitsuo Shiroma, Marconi de Arruda Pereira, Rone \\ Ilídio da Silva, Cristiano Maciel da Silva
}

\author{
Departamento de Tecnologia - Campus Alto Paraopeba \\ Universidade Federal de São João Del Rei - Ouro Branco - MG \\ \{sergiool, pshiroma, marconi, rone, cristiano\}@ufsj.edu.br
}

\begin{abstract}
Mobile Sinks has been used in Wireless Sensor Networks (WSNs) to increase network lifetime, especially to distribute, in homogeneous way, the energy consumption among all nodes. The development of low cost multicopters, aerial vehicles capable of autonomous flights, turn this type of vehicle a good solution to be used as mobile sinks. This work presents a new approach to the problem of how to define multicopter tours to collect data in WSNs, in order to extend the network lifetime. We consider a drone that can stop in a fixed predefined number of points inside a monitored area to collect sensor data. We propose a genetic model that shows how the distribution of the nodes' workload can extend the network lifetime. Simulation were performed to adjust the parameters of the model and to evaluate its performance.
\end{abstract}

Resumo: Sorvedouros móveis têm sido usados em Redes de Sensores Sem Fios (RSSF) visando aumentar o tempo de vida da rede, especialmente por distribuir, de forma mais homogênea, o consumo de energia por todos os nós. $O$ desenvolvimento de drones multirotores, veículos aéreos não-tripulados capazes de voos autônomos, de baixo custo, os tornam aptos a serem usados como sorvedouros móveis. Este trabalho considera que o drone pode parar em um número fixo de pontos da rede, característica dos multirotores, coletando dados de sensoriamento em um número fixo predefinido de pontos da rede. Este trabalho propõe um modelo genético que mostra como a distribuição da carga de trabalho dos nós pode estender o tempo de vida da rede. Simulações foram executadas para ajustar os parâmetros do modelo e avaliar seu desempenho.

\section{Introdução}

Redes de Sensores Sem Fio (RSSF) são redes ad hoc compostas por nós sensores espalhados em uma área geográfica de monitoramento, com o objetivo de coletar dados diversos e encaminhar esses dados para os nós sorvedouros. O maior problema das RSSF é prolongar o tempo de vida da rede explorando todas as suas funcionalidades. Nos últimos anos, foram apresentadas várias abordagens para o uso de sorvedouros móveis em RSSF visando o aumento do tempo de vida da rede. Visto que os nós mais próximos do sorvedouro tendem a apresentar um consumo maior de energia por sua função de roteamento, eles tendem a se exaurir mais rapidamente na configuração de sorvedouro fixo. O uso de sorvedouros móveis possibilita reduzir essa sobrecarga, distribuindo a função de roteamento pela rede (MA et al, 2013), (BASAGNI 
et al, 2008) e (WANG et al, 2014). Para tanto, é preciso identificar o melhor caminho e posicionamento para a coleta de dados.

Esse trabalho apresenta uma proposta que considera: i) a possibilidade de que o sorvedouro móvel possa parar sobre os pontos de coleta e aguardar que os dados se sensoriamento da região sejam enviados até ele; ii) os nós sorvedouros móveis tem energia limitada para percorrer a área monitorada; iii) os nós sensores tem energia limitada e posições, previamente conhecidas, e gastem energia de forma previsível, em função do número de mensagens transmitidas; iv) o raio de comunicação é simétrico e igual para todos os sensores. A partir destas premissas, este trabalho visa identificar qual o melhor posicionamento para que o drone pare e faça a coleta de dados, a cada viagem, até a exaustão da energia da rede, visando maximizar a mesma. Para tanto, apresenta um método para encontrar os melhores pontos de parada através do uso de algoritmos genéticos, visando maximizar o tempo de vida da rede.

Os resultados indicam que é possível estender o tempo de vida da rede significativamente. Comparando com as soluções aleatórias, o incremento no tempo de vida chega a $200 \%$. As soluções obtidas também foram comparadas em função no número de gerações genéticas, representadas por conjuntos de operações do algoritmo genético, indicando um aumento de cerca de $20 \%$ após algumas centenas de gerações genéticas. Também foram realizadas comparações com soluções baseadas em centroides. Os resultados, para essas comparações, indicaram a necessidade de uma energia inicial até $500 \%$ maior para manter o mesmo tempo de vida da rede em ambas as simulações.

O artigo está organizado da seguinte forma: a seção 2 apresenta os trabalhos relacionados. A seção 3 formaliza o problema. A seção 4 apresenta a estratégia de solução. A seção 5 apresenta os resultados. A seção 6 conclui o documento.

\section{Trabalhos Relacionados}

A maioria dos trabalhos considerados baseia-se somente no custo de comunicação entre nós sensores em suas abordagens, partindo do pressuposto que maximizar o tempo de vida da rede é equivalente a minimizar o consumo de energia (ROMAO et al. 2013, TASHTARIAN et al., 2012; ALMI'ANI et al, 2010). Embora esta correlação exista, este não é o único fator envolvido, também é preciso considerar a energia residual dos nós e a distribuição do consumo pelos nós da rede ao longo do seu tempo de vida. Existem alguns poucos trabalhos que consideram a energia residual nos nós sensores (LUO e JEAN-PIERRE, 2005; LIANG et al., 2011).

Embora o problema considerado seja NP-difícil, muitas vezes é possível compará-lo com o ótimo para pequenas instâncias. Normalmente ele é formulado como um problema de programação linear inteira (ALMI'ANI et al., 2010; LIANG et al., 2011; MA et al., 2013). Uma exceção é o trabalho de Luo e Jean-Pierre (2005) onde se resolve analiticamente o problema, encontrando a solução exata para o problema de determinação da trajetória do sorvedouro móvel. Entretanto, tal solução só é válida se os nós estiverem distribuídos em torno de um círculo de raio conhecido e seguindo uma distribuição de Poisson. Em seguida, uma heurística é utilizada para encontrar uma solução para o problema de roteamento.

SHI e HOU (2012) apresentam uma modelagem matemática para o problema de sorvedouro móvel em redes de sensores sem fio. Entre as contribuições, há uma redução 
do problema, com uma transformação tempo para espaço, de forma a tratar o problema apenas no domínio do espaço, ao invés de tratá-lo no domínio do tempo e espaço. Essa redução tem por objetivo facilitar o processo de otimização. Além disso, mostraram também que é possível discretizar o espaço de soluções, tornando-o finito, visto que, a princípio, seria infinito. Essas transformações aproximam-se do nosso trabalho e premissas, visto que, para o drone multirrotor, a autonomia é função do tempo de voo e não da distância percorrida.

Diversas heurísticas foram propostas para resolver o problema nos casos onde o número de nós sensores é grande o suficiente para invalidar a busca por uma solução exata. ALMI'ANI et al. (2010) propõem uma clusterização usando o algoritmo k-means para definir os pontos de coleta. Embora tal clusterização seja um bom critério para minimizar a quantidade média de saltos na rede como um todo, dependendo da topologia da rede é possível que o k-means crie clusters desbalanceados, deixando poucos nós com um número elevado de saltos que podem, consequentemente, causar a desconexão de um número significativo de nós.

ROMAO et al. (2013) propõem um algoritmo genético para determinar o conjunto de líderes de grupo que concentrarão os dados coletados, o roteamento de cada nó sensor para o respectivo líder de grupo, e um caminho visitando todos os líderes de grupo com tamanho menor que o máximo estabelecido. Para evitar um número excessivo de cálculos, o algoritmo genético foca apenas na seleção de quais nós sensores serão considerados como líderes de grupo, enquanto as outras etapas são feitas posteriormente.

Alguns trabalhos da literatura demandam prévio conhecimento, pelos nós, do caminho dos sorvedouros móveis (WANG et al, 2014), considerando que o sorvedouro não pode parar até que os dados cheguem até ele. Visto que o controle de posicionamento dos VANTs é eficaz, esses veículos são capazes de parar no ar com precisão para realizar a coleta dos dados de todos os nós de uma região específica. Diferenciam-se, ainda, de outros (SILVA e NASCIMENTO, 2016) por ter como objetivo maximizar o tempo de vida da rede e considerar isso no modelo.

Este trabalho desenvolve uma solução integrada onde o roteamento dos pacotes na RSSF e a determinação das trajetórias dos sorvedouros móveis são tratados de forma conjunta, e não isoladamente. A trajetória do sorvedouro móvel é restrita apenas pela autonomia de voo do drone e os pontos de coleta escolhidos podem estar em qualquer posição do espaço. O roteamento dos pacotes considera não apenas o custo de comunicação entre nós, mas também a energia residual.

\section{Formalização do Problema}

A rede de sensores sem fio com sorvedouro móvel pode ser representa pela tupla $(S, \mathrm{~A}$, $r, m)$, no qual:

- $S=\left\{s_{1}, s_{2}, s_{3}, \ldots, s_{n}\right\}$, o conjunto de $n$ nós sensores, espalhados de forma aleatória sobre a área sensoriada;

- $A$, a área monitorada, quadrada, com lado de L metros;

- $\quad r$ - alcance do rádio dos nós sensores, fixo e idêntico para todos os nós; 
- $\quad m$ - número de posições que o sorvedouro móvel assume para coletar dados.

O resultado do problema deve indicar uma sequência $J$, ordenada, de conjuntos $D_{i}$, de tamanho $m$, os quais representam as posições assumidas pelo sorvedouro móvel a cada passada $i$, de forma a maximizar o tempo de vida da rede, indicado como o momento em que $1 \%$ dos nós da rede tem sua bateria exaurida (ALMI'ANI et AL, 2010).

O problema consiste em encontrar a melhor sequência $J$, que resulta no tempo de vida máximo para a rede. Esse problema envolve um número infinito de soluções, visto que o drone pode parar em qualquer ponto na área dentro da qual se encontram os nós sensores. Uma possível discretização dos pontos candidatos a receber o drone, que tornaria os conjuntos de possíveis soluções finitos, pode ser modelada pelo Problema da Mochila (Knapsack problem), notadamente NP completo.

\section{Estratégia de Solução}

Algoritmos genéticos são algoritmos evolucionários, isto é, partem de uma população de soluções viáveis (indivíduos) para o problema, onde cada solução é construída de forma aleatória. Essa população é submetida a operações genéticas (cruzamento, mutação e seleção) de forma que aqueles indivíduos mais aptos (apresentam maior adequação ao problema) são selecionados para as próximas gerações, além de serem mais utilizados nas operações de cruzamento. A avaliação do desempenho do indivíduo no problema (i.e. o grau de "aptidão") é medido pela função fitness. Assim, quando se utiliza um algoritmo genético, faz-se necessário identificar quais são os aspectos mais relevantes da solução a serem considerados, de tal maneira a serem otimizados pelo algoritmo em questão. Particularmente neste trabalho, algoritmos evolucionários podem melhorar a solução final de problemas de posicionamento do sorvedouro móvel em redes de sensores, desde que se conheça previamente o estado atual dos nós, tanto em termos de posicionamento quanto energia.

A modelagem do problema para que seja solucionado com um algoritmo genético deve considerar o conceito de população, que representa um conjunto de soluções viáveis (possíveis) para o problema. Cada solução viável é chamada de indivíduo; a avaliação da população se dá pela avaliação de cada indivíduo, pela qual cada uma dessas soluções viáveis é avaliada com uma pontuação, que representa a qualidade da solução para o problema. $\mathrm{O}$ valor dessa avaliação serve de base para que um indivíduo participe de operações genéticas como mutação e cruzamento.

A população, para este problema, será representada por um conjunto de soluções instantâneas para o posicionamento do sorvedouro móvel. Cada solução permitirá determinar qual o melhor posicionamento para o sorvedouro móvel para a configuração atual da rede, incluindo sua disponibilidade de energia e conectividade. A cada ciclo de sensoriamento, o algoritmo genético será executado, visando identificar os pontos de parada do drone para coletar os dados de sensoriamento. A avaliação da qualidade da solução vai considerar, prioritariamente, o impacto da solução no tempo de vida da rede. Essa avaliação vai considerar a energia gasta no roteamento e a energia residual dos nós após o processo. Os nós com menos energia devem ser poupados da função de roteamento, garantindo seu funcionamento por mais tempo. 
A operação de mutação consistirá no deslocamento de um ponto de coleta do sorvedouro móvel, gerando uma solução diferente da solução usada para a mutação. O deslocamento será de um valor aleatório entre zero e o alcance do rádio, intervalo que se espera alterações efetivas na solução, visto que deslocamentos pequenos não alteram a solução. Vale destacar que os indivíduos resultantes da operação de mutação continuam válidos, isto é, continuam como uma solução viável para o problema. A operação de cruzamento será realizada considerando um particionamento geográfico dos pontos de coleta. As novas soluções são geradas tomando metade dos pontos de coletas mais à direita de uma solução e metade dos pontos de coleta mais à esquerda de outra solução.

\subsection{Exaustão da energia da rede}

Após definida a modelagem, o algoritmo genético é executado de forma exaustiva, até exaurir a energia de $1 \%$ dos nós da rede, considerado então o limite para o tempo de vida. O conjunto solução, nesse caso, inclui configurações diversificadas para o posicionamento dos sorvedouros móveis a cada fase de coleta, pois, do contrário, os nós próximos ao sorvedouro móvel teriam sua energia exaurida mais rapidamente.

\subsection{Balanceamento da energia}

Dois princípios foram aplicados para balancear a energia da rede e, consequentemente, prolongar o tempo de vida na rede: 1) roteamento dos pacotes por caminhos de maior energia residual; e 2) consumo mínimo de energia para coleta dos dados. Estes princípios foram usados em dois momentos: 1) na forma como os nós encaminham os dados até o sorvedouro móvel; e 2) como é calculada a função fitness. Para tanto, dois parâmetros foram considerados: a energia gasta na fase de coleta e a distribuição da energia residual dos nós. A relação entre a energia gasta e a energia residual em cada fase de coleta deve ser tal que a energia residual deve ser tão balanceada quanto possível após cada fase de coleta.

A escolha do caminho a ser percorrido, considerou também o custo de execução na definição do roteamento, de forma a criar uma solução já balanceada, isto é, evitando sobrecarregar um ou mais nós da rede, de tal maneira a evitar que esses nós se tornassem um gargalo. A disponibilidade de outro caminho com maior energia residual faz com que ele seja escolhido para o encaminhamento dos pacotes, resultando em uma solução mais balanceada. A Figura 1 apresenta um exemplo do funcionamento da escolha do caminho para o roteamento.

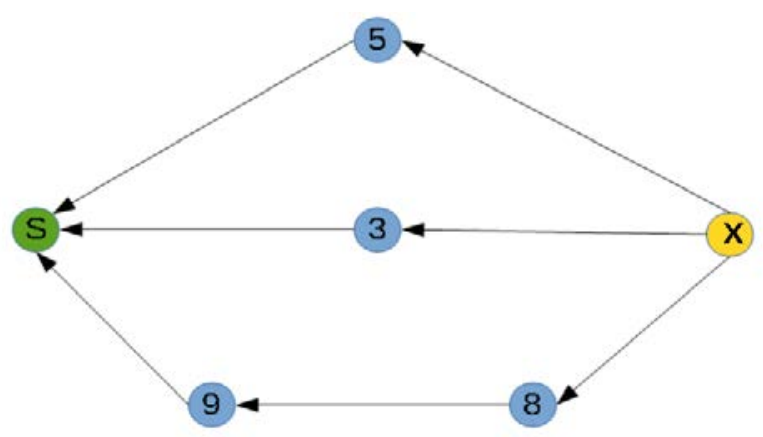

Figura 1 - Exemplo de balanceamento de energia no roteamento.

A escolha da formulação adequada para definir as rotas, bem como a função fitness, iniciou-se somando o custo relativo de cada salto do roteamento, definido como 
a relação entre o custo do salto, sempre considerado como uma unidade, e sua energia residual $\left(1 / \mathrm{E}_{\mathrm{i}}\right)$. Nas primeiras simulações usando essa relação, foi percebida a importância de reforçar a energia residual nessa formulação, razão pela qual foi atribuído, a ela, um fator denominado fator de apreciação da energia residual $\alpha$, chegando então à soma dos custos relativos elevados ao fator $\alpha:\left(1 / \mathrm{e}_{\mathrm{i}}\right)^{\alpha}$. Para o cálculo da função fitness, simplesmente foram somados todos os custos relativos de transmissão de todos os nós da rede, resultando na seguinte formulação:

$$
F_{i}=\sum_{j=0}^{n} \frac{e_{j}}{E_{j i}^{\alpha}}
$$

Onde: $F_{i}$ é o resultado da função de avaliação fitness após a fase de coleta $i$, com $n$ nós sensores, $e_{j}$ representa a energia gasta pelo nó $j$ na fase $i$ e $E_{j i}$ representa a energia residual do nó $j$ após a fase $i$.

Encontrar o melhor valor de $\alpha$ que otimizasse o problema aqui apresentado passou a ser parte do problema, de forma que foram executadas várias simulações com esse objetivo, como pode ser visto na seção 5. Foi identificado que $\alpha=3$ apresenta bons resultados para a heurística proposta.

Para melhor entendimento, seja o exemplo da Figura 1. Supondo o nó $S$, como nó sorvedouro, ponto onde o drone faz a coleta de dados, e o nó $\mathrm{X}$ a ser inserido na coleta, ele tem três possibilidades de encaminhamento de dados ao sorvedouro. Em cada nó, está representada sua energia residual. Na primeira alternativa, superior, como a energia residual do nó é 5 , o custo desse caminho é $1 / 5^{3}$, igual a 0,008 . Na segunda, pelo nó com energia residual 3 , o custo é $1 / 3^{3}$, igual a 0,037 ; e, finalmente, na última, o custo seria $1 / 9^{3}+1 / 8^{3}$, resultando 0,003 ; se tornando, então, a melhor solução, apesar do caminho utilizar de duas unidades de energia para realizar os dois saltos até o sorvedouro.

\section{Resultados}

Usando o ambiente de programação Anaconda para a linguagem Python, foi implementado um simulador usando o modelo genético para obter os resultados esperados para este trabalho.

Como massa de testes, foram gerados vários arquivos, variando o número de nós de 50 a 1000, e variando algum parâmetro de entrada, como a função fitness, número de nós e número de gerações para o modelo genético. As simulações foram executadas 30 vezes, tomando a média e o desvio padrão, que só não foi registrado neste artigo na seção 5.1, dado o grande volume de dados a ser apresentado.

\subsection{Avaliando a Energia por Fase}

Com o objetivo de verificar se cada fase de coleta tem um consumo de energia compatível com outras soluções, um conjunto de simulações foi realizado, verificandose apenas a energia média gasta pelos nós para aquela fase. Foram avaliados uma distribuição uniforme de nós "Grid", além dos algoritmos de localização de centroides conhecido como K-Means (ALMI'ANI et al., 2010), e o modelo genético proposto neste trabalho com 20, 40 e 100 gerações. 
A Figura 2 apresenta os resultados. Como apresenta a energia média para apenas uma fase de coleta, os valores são próximos, mas é possível identificar uma ordem decrescente de energia: grid, genético com 20 gerações, K-Means, genético com 40 gerações e genético com 100 gerações.

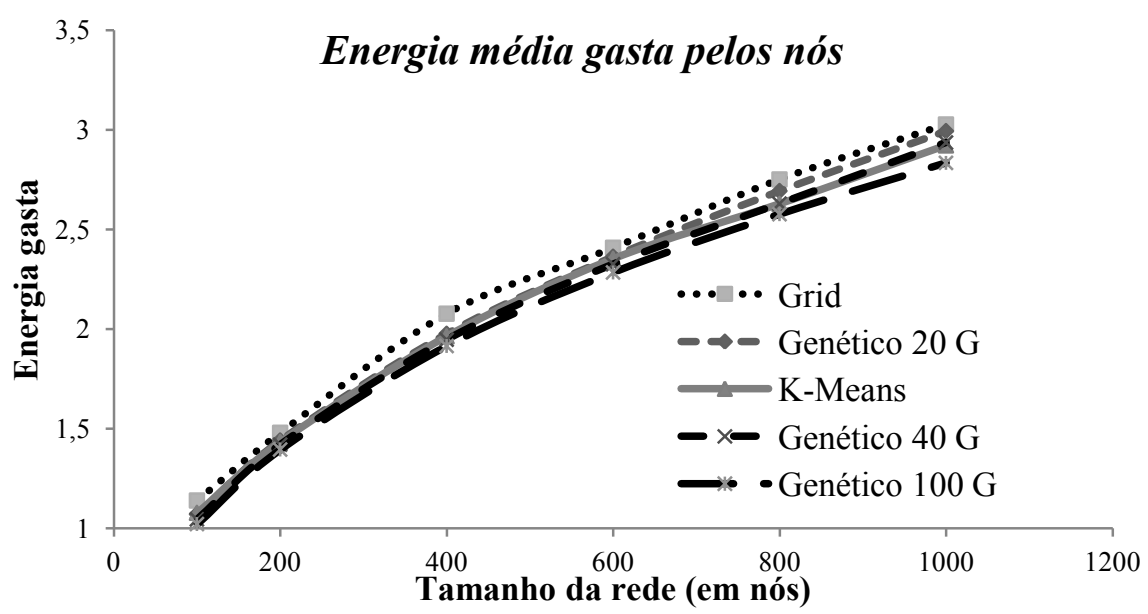

Figura 2 - Comparação da energia gasta com outras soluções

O modelo genético utilizado está configurado para balancear a energia da rede e não apenas minimizar o consumo de energia para uma única fase de coleta. Essa simulação teve por objetivo apenas mostrar que a solução obtida é compatível com outras soluções. É possível configurar o algoritmo genético para minimizar apenas a energia consumida em uma fase única, o que resultaria em resultados melhores, mas não faz parte dos objetivos deste trabalho.

\subsection{Cálculo da Função Fitness}

A próxima simulação foi realizada com o objetivo de identificar qual seria a melhor função fitness. Essa função deverá maximizar o tempo de vida da rede, reduzindo o consumo dos nós e o promovendo de forma balanceada. Partindo da consideração que cada transmissão consuma sempre uma unidade de energia e que a manutenção da energia residual torne sua conservação fundamental, foi escolhida a função $1 / E^{\alpha}$, sendo $E$ a energia residual do nó, como custo relativo de cada transmissão. Para essa simulação foram considerados 50 nós, em uma área 1000x1000 e a execução ocorreu até o limite definido para o tempo de vida da rede.

Tabela 1 - Resultados da seleção da função fitness

\begin{tabular}{|c|c|c|}
\hline Função fitness & Média & Desvio Padrão \\
\hline $1 / \mathrm{x}$ & 29,30 & 0,92 \\
\hline $1 / \mathrm{x}^{2}$ & 32,87 & 0,63 \\
\hline $1 / \mathrm{x}^{3}$ & 33,80 & 0,61 \\
\hline $1 / \mathrm{x}^{4}$ & 33,33 & 0,88 \\
\hline $1 / \mathrm{x}^{5}$ & 33,40 & 0,86 \\
\hline
\end{tabular}

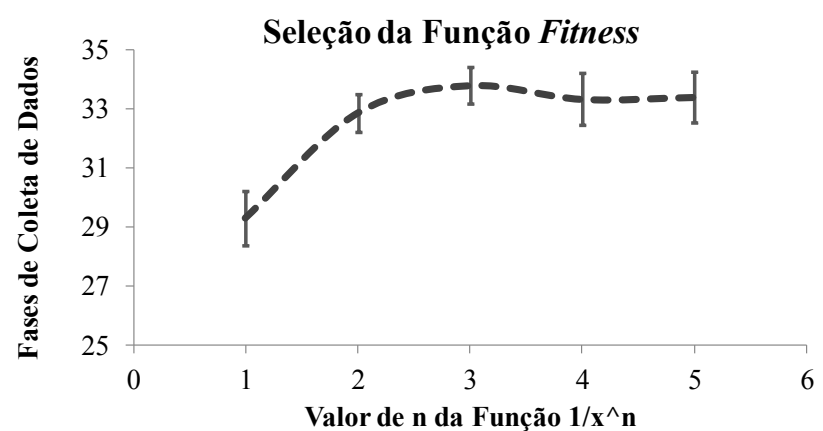

Figura 3 - Resultados da seleção da função fitness 
A tabela 1 apresenta os resultados obtidos. A figura 3 apresenta os resultados graficamente. Observa-se que a função $1 / \mathrm{x}^{3}$ apresenta os melhores resultados para a massa de testes considerada, com pequena variação para as funções $1 / \mathrm{x}^{2}, 1 / \mathrm{x}^{4} \mathrm{e} 1 / \mathrm{x}^{5}$, que também poderiam ser consideradas. A partir da escolha da função, iniciamos os testes com o objetivo de validar a modelagem genética como método para alcançar uma solução boa para o problema de escolher o posicionamento do sorvedouro móvel.

\subsection{Estendendo o tempo de vida da rede}

O principal resultado esperado nos testes foi o número de operações de sensoriamento e coleta que a rede poderia fazer durante seu ciclo de vida. Foram realizados dois conjuntos de simulações com essa finalidade.

O primeiro conjunto tem por objetivo comparar o modelo genético com outras soluções e, para tanto, foi usada como referência a implementação do $k$-means, como em ALMI'ANI et al. (2010). Para esse primeiro conjunto, o número de nós foi usado como parâmetro, variando entre 100 e 500 nós, mas mantendo fixa a densidade de nós por área, ou seja, a área foi aumentada proporcionalmente ao número de nós para manter a conectividade média, como pode ser visto na figura 4 .

\begin{tabular}{|c|c|c|}
\hline \multirow{2}{*}{\multicolumn{3}{|c|}{ K-Means }} \\
\hline & & \\
\hline $\mathrm{N}$ & Média & $\begin{array}{l}\text { Desvio } \\
\text { Padrão }\end{array}$ \\
\hline 100 & 12,00 & 1,08 \\
\hline 200 & 46,70 & 9,60 \\
\hline 300 & 92,20 & 10,17 \\
\hline 400 & 108,63 & 12,49 \\
\hline 500 & 152,73 & 23,02 \\
\hline \multicolumn{3}{|c|}{ Genético $40 \mathrm{G}$} \\
\hline $\mathrm{N}$ & Média & $\begin{array}{l}\text { Desvio } \\
\text { Padrão }\end{array}$ \\
\hline 100 & 3,13 & 0,43 \\
\hline 200 & 12,50 & 1,81 \\
\hline 300 & 18,63 & 2,41 \\
\hline 400 & 26,23 & 3,22 \\
\hline 500 & 34,67 & 3,92 \\
\hline
\end{tabular}

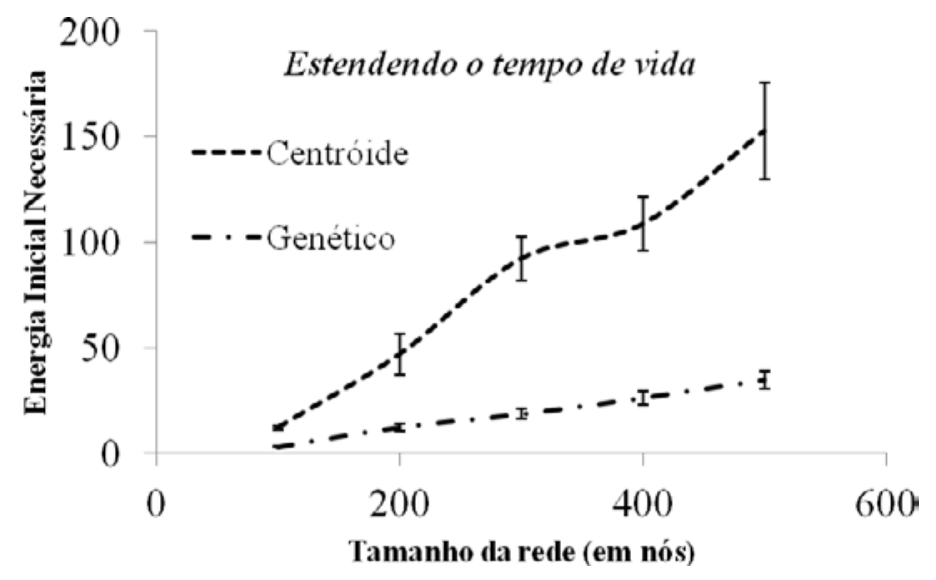

Figura 4 - Energia necessária para tempo de vida

O segundo conjunto tem por objetivo avaliar como o número de gerações do algoritmo genético interfere na qualidade da solução encontrada. Para tanto, foi considerada uma simulação com o número fixo de 50 nós, em uma área 1.000 x 1.000, 4 pontos de parada para o nó sorvedouro e energia inicial igual a 100 unidades, executando até que os parâmetros de fim do tempo de vida da rede sejam alcançados (exaustão de energia de $1 \%$ dos nós da rede), como visto na Figura 5.

Foi adotado o princípio de que, quanto maior a quantidade de gerações do algoritmo genético, mais a solução encontrada se aproximam da solução ótima. O caso base considerado foi um caso no qual nenhuma operação genética havia sido realizada, e o posicionamento do sorvedouro móvel é realizado de forma aleatória.

Observa-se uma melhora significativa do tempo de vida da rede, em mais de 10 fases de sensoriamento, comparando com uma primeira solução com apenas uma 
geração de operações genéticas, para uma energia inicial de 100 unidades. Comparando com a solução aleatória, o ganho é de quase $200 \%$, registrada na Tabela 3 como "zero" gerações genéticas.

Tabela 3 - Tempo de vida
em função do número de
gerações
\begin{tabular}{|c|c|c|}
\hline Gerações & Média & $\begin{array}{c}\text { Desvio } \\
\text { padrão }\end{array}$ \\
\hline 0 & 27,53 & 3,95 \\
\hline 1 & 63,27 & 1,01 \\
\hline 2 & 64,17 & 0,87 \\
\hline 4 & 65,13 & 1,01 \\
\hline 8 & 66,63 & 0,81 \\
\hline 16 & 68,83 & 0,83 \\
\hline 32 & 70,90 & 0,71 \\
\hline 64 & 72,43 & 0,73 \\
\hline 128 & 73,77 & 0,73 \\
\hline 256 & 74,43 & 0,63 \\
\hline
\end{tabular}

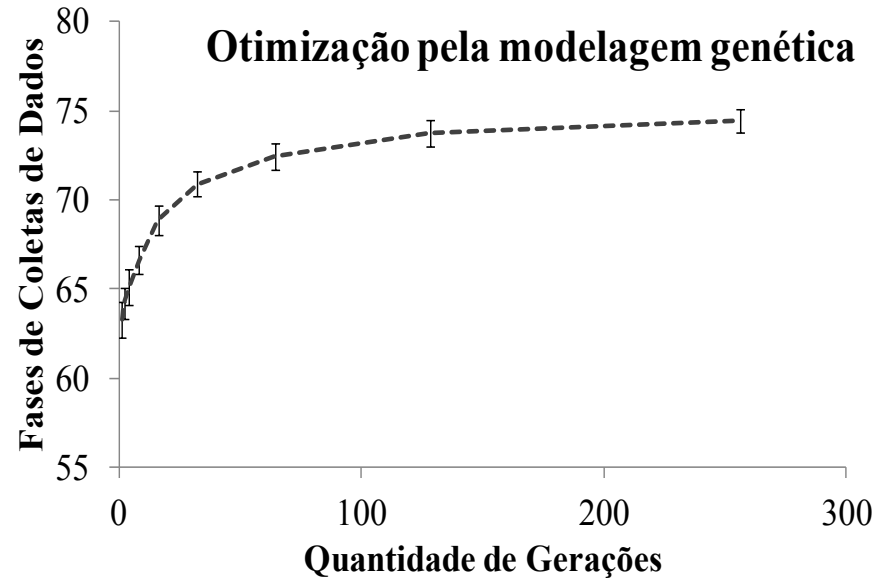

Figura 5 - Tempo de vida em função do número de gerações

Observa-se, ainda, que o tempo de vida da rede estabiliza-se próximo a 75 fases de coleta, indicando um possível limite de uma solução ótima. Considerando as 100 unidades de energia dos nós, o custo de uma unidade para o repasse de cada mensagem e a necessidade de encaminhar algumas mensagens em múltiplos saltos para atingir alguma posição do sorvedouro, essa solução pode ser considerada um resultado bem próximo do esperado.

O máximo teórico de 100 fases só poderia ser atingido se a cobertura do alcance do sorvedouro incluísse todos os nós em comunicação direta, o que não é possível para o exemplo citado, bem como não representaria um problema a ser tratado, visto que, nesse caso, seria fácil obter a solução ótima, na qual todos os nós enviam diretamente ao sorvedouro e o tempo de vida seria igual à energia inicial dos nós.

\section{Conclusões e Trabalhos Futuros}

Este artigo apresentou a implementação de uma modelagem genética para localizar os pontos de parada de um sorvedouro móvel, implementado por um drone, em uma rede de sensores sem fio, visando equilibrar o consumo de energia da rede e, assim, prolongar o tempo de vida da rede. A abordagem utilizada se difere das principais referências da literatura por considerar um drone multirrotor, capaz de permanecer em uma determinada posição pelo tempo necessário por realizar a coleta dos nós da região onde se encontra.

O deslocamento do drone entre os pontos de parada foi desprezado, uma vez que ele pode ser feito na velocidade máxima de deslocamento do drone, que tem sua autonomia dada em tempo, e não em distância percorrida. Assim, a limitação imposta foi o número de paradas do drone. Os resultados mostram que a modelagem genética é uma boa alternativa para definir os pontos de parada do drone, visando maximizar o consumo de energia da rede. Definindo a função fitness de forma a balancear a energia da rede, é possível estender o tempo de vida de forma a exaurir toda a energia da rede uniformemente. Comparando com outras soluções não balanceadas, como K-Means, foi 
possível identificar um aumento do tempo de vida da rede em algumas vezes, identificando, aí, a maior contribuição deste trabalho.

Como trabalho futuro é possível obter um método alternativo para o cálculo da solução ótima para uma instância mais reduzida de testes, para comparar com a solução genética, com o objetivo de apurar a proximidade da solução alcançada com a solução ótima. Também é interessante considerar nós sensores capazes de variar a potência do rádio durante a transmissão, visando economizar energia nas transmissões para os nós mais próximos. Essa abordagem apresentaria resultados interessantes, visto que privilegiaria transmissões para nós mais próximos, ao mesmo tempo em que deve privilegiar nós com maior disponibilidade de energia.

\section{Referências}

BASAGNI, S., et al. Controlled sink mobility for prolonging wireless sensor networks lifetime. Wireless Networks, vol. 14, no. 6, pp. 831-858, 2008.

ALMI'ANI, J. et al. Energy-efficient data gathering with tour length-constrained mobile elements in wireless sensor networks. In: IEEE CONFERENCE ON LOCAL COMPUTER NETWORKS, 2010, Denver. pp.582-589.

LIANG, W.; LUO, J. Network lifetime maximization in sensor networks with multiple mobile sinks. In: IEEE CONF. ON LOCAL COMPUTER NETWORKS, 2011, Bonn. pp.350-357.

LUO, J.; JEAN-PIERRE, H. Joint mobility and routing for lifetime elongation in wireless sensor networks. In: IEEE CONFERENCE ON COMPUTER COMMUNICATIONS (INFOCOM), 2005, Miami. pp.1735-1746.

MA, M. et al. Tour planning for mobile data-gathering mechanisms in wireless sensor networks. IEEE Trans. Vehicular Technol, vol 62, no. 4, 1472-1483, 2013.

ROMAO, O. C. et al. Lifetime maximization of hop-and-delay constrained wireless sensor networks with mobile agents. In: IEEE CONGRESS ON EVOLUTIONARY COMPUTATION (CEC), 2013. pp.1083-1090.

SHOLES, E. Evolution of a UAV Autonomy Classification Taxonomy. In: IEEE Aerospace Conference, Big Sky, MT, 2007

SHI, Y. e HOU, Y. T. Some Fundamental Results on Base Station Movement Problem for Wireless Sensor Networks. IEEE/ACM Transactions on Networking, vol. 20, no.4, pp.1054-1067, Aug. 2012

SILVA, R. I. e NASCIMENTO, M. A. - "On best drone tour plans for data collection in wireless sensor network," in Proceedings of the 31st ACM Symposium on Applied Computing, SAC 2016, Pisa, Italy, April 4-8, 2016, 2016.

TASHTARIAN, F. et al. Energy efficient data gathering algorithm in hierarchical wireless sensor networks with mobile sinks. In: INTERNATIONAL CONFERENCE ON COMPUTER AND KNOWLEDGE ENGINEERING, 2012. pp.350-357.

WANG, J. et al. A Survey about Routing Protocols with Mobile Sink for Wireless Sensor Network. International Journal of Future. Generation Communication and Networking, vol.7, no.5, pp.221-228, 2014. 